\title{
Creating nanoporous graphene with swift heavy ions
}

\author{
H. Vázquez ${ }^{\mathrm{a}}$, E. H. Åhlgren ${ }^{\mathrm{a}, \mathrm{b}}$, O. Ochedowskic ${ }^{\mathrm{c}}$, A. A. Leino ${ }^{\mathrm{a}}$, R. \\ Mirzayev $^{\mathrm{d}}$, R. Kozubek ${ }^{\mathrm{c}}$, H. Lebius ${ }^{\mathrm{f}}$, M. Karlušic ${ }^{g}$, M. Jakšic ${ }^{g}$, A. V. \\ Krasheninnikov $^{\text {he,i, }}$, J. Kotakoskid ${ }^{\mathrm{d}}$, M. Schleberger ${ }^{\mathrm{c}}$, K. Nordlund ${ }^{\mathrm{a}}$, F. \\ Djurabekova ${ }^{\mathrm{a}, *}$ \\ ${ }^{a}$ Department of Physics, University of Helsinki, P.O. Box 43, 00014 Helsinki, Finland \\ ${ }^{b}$ School of Chemistry, University of Nottingham, University Park, Nottingham NG7 \\ 2RD, United Kingdom \\ ${ }^{c}$ Fakultät für Physik and CENIDE, Universität Duisburg-Essen, 47048 Duisburg, \\ Germany \\ ${ }^{d}$ Fakultät für Physik, Universität Wien, Boltzmanngasse 5, 1090 Vienna, Austria \\ ${ }^{e}$ Department of Applied Physics, Aalto University, P.O. Box 1100, 00076 Helsinki, \\ Finland \\ ${ }^{f}$ CIMAP, (CEA-CNRS-ENSICAEN-UCN), blvd Henri Becquerel, 14070 Caen, France \\ ${ }^{g}$ Ruder Boskovic Institute, Bijenicka cesta 54, 10000 Zagreb, Croatia \\ ${ }^{h}$ Helmholtz-Zentrum Dresden-Rossendorf, Institute of Ion Beam Physics and Materials \\ Research, 01328 Dresden, Germany \\ ${ }^{i}$ National University of Science and Technology MISiS, 4 Leninskiy prospekt, Moscow, \\ 119049, Russian Federation
}

\begin{abstract}
We examine swift heavy ion-induced defect production in suspended single layer graphene using Raman spectroscopy and a two temperature molecular dynamics model that couples the ionic and electronic subsystems. We show that an increase in the electronic stopping power of the ion results in an increase in the size of the pore-type defects, with a defect formation threshold at $1.22-1.48 \mathrm{keV} /$ layer. We also report calculations of the specific electronic heat capacity of graphene with different chemical potentials and discuss the electronic thermal conductivity of graphene at high electronic temperatures, suggesting a value in the range of $1 \mathrm{Wm}^{-1} \mathrm{~K}^{-1}$. These results indicate that swift heavy ions can create nanopores in graphene, and that their size can be tuned between 1-4 $\mathrm{nm}$ diameter by choosing a suitable stopping power.
\end{abstract}

\footnotetext{
*Corresponding author.

Email address: flyura.djurabekova@helsinki.fi (F. Djurabekova)
} 


\section{Introduction}

Graphene has many attractive properties which have lead scientists to seek ways to apply this material. For example, it has been used in DNA sequencing [1], chemical sensing [2], gas separation [3, 4, 5] and water desalination [6]. Many of these applications require post-synthesis atomic scale modification of the material, which can not be accomplished by directly using the conventional methods without adapting them first for nanomaterials. For example, ion implantation of graphene requires energies several orders of magnitude lower than the conventional three-dimensional materials used in the semiconductor industry $[7,8,9,10,11]$. Also other types of modifications are possible with ion beams $[12,13,14,15,16]$, but the effects depend sensitively on ion energy.

A rough division can be made between low energy ion irradiation (a few $\mathrm{keV} /$ nucleon) and high energy irradiation (a few hundreds of $\mathrm{keV} /$ nucleon). The ions in the latter energy range are referred to as swift heavy ions or SHI [17]. The lower energy ions slow down in materials via atomic collisions, whereas the high energy ions lose energy via interactions with the electronic subsystem, causing ionization and electronic excitations along the ion path. These channels of energy loss are characterized by nuclear $\left(S_{n}\right)$ and electronic $\left(S_{e}\right)$ stopping powers, respectively, and are expressed in units of energy loss/length.

Although many studies [18, 19, 20, 21, 22, 23] have addressed the underlying mechanism of defect formation by SHI, these mechanisms still remain unclear. Several hypotheses have been proposed, such as Coulomb explosion [18, 19], inelastic thermal spike [20, 21], exciton self-trapping [22] and combinations of these $[23,24]$. Regardless of the underlying mechanism, it is well established that SHI tend to create structural modifications with cylindrical symmetry ('tracks') along the ion path within the target material [25].

Only a few experimental studies have addressed the effect of SHI irradiation in graphene [26, 27, 28, 29, 30, 31, 32, 33]. Most of these experiments were performed on supported graphene and under oblique incidence. Measurement of SHI induced defects in single layers of suspended graphene under perpendicular incidence is still a challenge. During the experiments, the graphene samples become covered with hydrocarbon contaminants, which effectively prevent imaging of the defects under the contamination atoms. This 
makes it impossible to verify the pore-like nature of the irradiation-induced defects with imaging methods. Atomistic simulations can assist understanding of the processes that take place during irradiation. However, the model describing the interaction of ions with electrons in the simulations is of crucial importance, since it defines the dynamics of energy exchange between the electronic and ionic sublattices of graphene and hence is responsible for formation of final defects. In recent molecular dynamics simulations by Zhao and Jianming [16], the interaction of SHI with graphene was described by the inelastic thermal spike model $[34,35,36]$, and the energy deposited on atoms by excited electrons was added instantaneously within a certain radius around the ion path. Although this model has proven sufficient for some cases in three-dimensional materials $[37,38,39,34,40,41]$, in two-dimensional materials as graphene it is oversimplified, since it does not take into account the electronic subsystem and its energy exchange with the lattice during the simulation. Moreover, due to these simplifications, the model may fail to capture the key processes developing in a single layer material, leading to a strong uncertainty in the damage formation threshold, reported as 5.3-8 $\mathrm{keV} / \mathrm{nm}$ (1.8-2.7 keV/layer) without validation from experiments.

In this study, we report a combined experimental and theoretical study of defect formation in suspended graphene by SHI ions. The induced defects are analyzed by Raman spectroscopy in combination with the two temperature molecular dynamics (TTMD) model [42], which couples the ionic and electronic subsystems in a concurrent multiscale simulation. This model was able to resolve inconsistency of experimental observations by SAXS and RBS measurements reported in [43] in contrast to the instantaneous thermal spike model [44]. In this study, we show that, assuming a constant electronic thermal conductivity of $1 \mathrm{Wm}^{-1} \mathrm{~K}^{-1}$, TTMD succeeds in reproducing the threshold for damage formation and the defect behaviour observed for graphene in two independent experiments. The good agreement between both experiments and the simulations establishes $1.22-1.48 \mathrm{keV} /$ layer as the defect formation threshold in graphene.

\section{Methods}

\subsection{Sample preparation and irradiation}

We report the results of two independent experiments carried out on suspended single layer graphene samples. For clarity, we will refer to the results of our two different experiments as set A and set B. The graphene 
samples of set A were prepared using the mechanical exfoliation technique of a graphite crystal onto a patterned $\mathrm{SiO}_{2} / \mathrm{Si}$ substrate. The pattern consisted of periodically etched holes in the sample with a diameter of $3.2 \mu \mathrm{m}$ and a depth of about $8 \mu \mathrm{m}$. Graphene covering these holes is suspended (see Fig. 1 (a)). The layer thickness was checked by Raman spectroscopy using the FWHM of the $2 D$ band [45]. In all samples, the disorder-induced $D$ band was absent prior to irradiation. For the samples of set A, a linear background subtraction was applied. The samples of set B were commercially purchased. Graphene was grown by the chemical vapor deposition method [46] and transferred onto gold transmission electron microscopy grids with a porous carbon mesh by the provider. In this set, the size of the holes of the mesh $(\sim 1 \mu \mathrm{m})$ was close to the size of the laser beam, so that in most cases the recorded spectra contained a contribution of the $D$ band. This background contribution was subtracted from the data presented in this work. For both sets A and B, the peaks were fitted with a combination of Gaussian and Lorentzian profiles with their spectral weight as a free parameter. Point spectra were taken from several different positions and then averaged. The error bars in figures 1 and 3 are the FWHM of the corresponding distribution.

The samples of set A were irradiated by SHI projectiles of varying kinetic energy at the IRRSUD beamline of GANIL (Caen, France) with $91 \mathrm{MeV}$ Xe, at the Tandem van de Graaff accelerator of the RBI (Zagreb, Croatia) with $23 \mathrm{MeV}$ I, $15 \mathrm{MeV}$ Si, $6 \mathrm{MeV} \mathrm{Si,} 3 \mathrm{Mev}$ O, and $1 \mathrm{MeV}$ O, while the samples of set $\mathrm{B}$ were irradiated at the Tandem van de Graaff accelerator TAMIA at the University of Helsinki (Finland) with $23 \mathrm{MeV}$ and $35 \mathrm{MeV} \mathrm{C,} 15 \mathrm{MeV}$ and $35 \mathrm{MeV} \mathrm{Si}$, and $35 \mathrm{Mev} \mathrm{Au}$ (see table 1). No stripping foil was used during irradiation. All samples were irradiated under perpendicular incidence at room temperature and with a constant fluence of either $50.000 \mathrm{SHI} / \mu \mathrm{m}^{2}$ (set A) or $100.000 \mathrm{SHI} / \mu \mathrm{m}^{2}$ (set B). These fluences were chosen to produce a sufficiently high signal-to-noise ratio, while both still having low overlapping of tracks. Therefore, in this regime we can safely assume that the ratio of the $\mathrm{D}$ and $\mathrm{G}$ bands does not yet change significantly with the fluence (see Ref. [32]), enabling us to compare the $\mathrm{D} / \mathrm{G}$ ratio as a function of $\mathrm{dE} / \mathrm{dx}$ at a fixed fluence. After irradiation under high vacuum conditions, the samples were transferred back to ambient conditions to be investigated by Raman spectroscopy. 

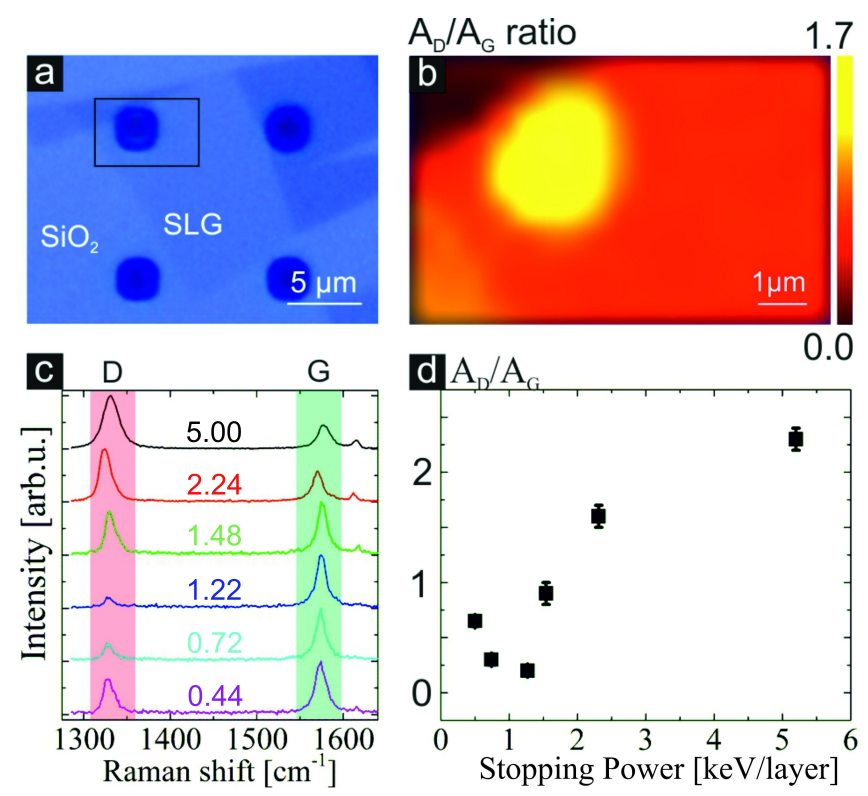

Figure 1: (a) Optical image of a graphene flake exfoliated on a patterned $\mathrm{SiO}_{2}$ substrate. (b) Raman mapping of the $\mathrm{A}_{D} / \mathrm{A}_{G}$ peak area ratio of the box marked in the optical image. This sample was irradiated with $23 \mathrm{MeV}$ I ions. The ratio is significantly enhanced for suspended graphene compared to $\mathrm{SiO}_{2}$ supported graphene. (c) Some of the measured Raman spectra showing the evolution of the $D$ (peaks marked in red) and the $G$ (peaks marked in green) bands for different stopping powers in keV/layer. The fluence was 50.000 ions $/ \mu \mathrm{m}^{2}$. (d) The corresponding $\mathrm{A}_{D} / \mathrm{A}_{G}$ peak area ratios plotted against the electronic stopping power of the SHI as calculated by SRIM. All results are shown for the graphene samples of set A (see text).

\subsection{Raman spectroscopy}

Raman spectroscopy is a common non-destructive method to study defects in graphene. The $D$ band (at $1350 \mathrm{~cm}^{-1}$ ), shown in Fig. 1 (c) for stopping powers equal or larger than $1.48 \mathrm{keV} /$ layer, is a direct consequence of the presence of defects in the graphene lattice [47, 48]. A quantitative defect analysis, i.e. the determination of their size and their nature, respectively, is however not an easy task. Lucchese et al. proposed a model based on the peak intensity ratios of the $D$ and $G$ peaks $\left(\mathrm{I}_{D} / \mathrm{I}_{G}\right)$, which can be used to determine the density of defects in a single layer graphene sheet, provided the defect size is known [49]. Vice versa, the size can be determined, in principle, if the defect density is known. The defect density in our experiment becomes equal to the fluence (50.000 ions $/ \mu \mathrm{m}^{2}$ for set $\mathrm{A}$ and 100.000 
ions $/ \mu \mathrm{m}^{2}$ for set B) if we assume an efficiency (probability of defect creation per ion) of one for all the ions. This assumption should hold for high $S_{e}$ ions, however, in the low $S_{e}$ range it may add uncertainties to our analysis. Because the specific type of the SHI induced defects is not known we cannot derive their size directly from the Raman data. We circumvent this problem by using the peak ratios only to determine the relative size of the defects induced by irradiations with different SHIs. Then, by comparing these results with the simulation results, we obtain information about the actual defect size for every ion type used in the experiments. Note, that we used the area ratios $\mathrm{A}_{D}$ and $\mathrm{A}_{G}$ (the areas underneath the $D$ and $G$ peaks, respectively) rather than the intensity ratios $\mathrm{I}_{D}$ and $\mathrm{I}_{G}$, because it has been recently shown that the $\mathrm{I}_{D} / \mathrm{I}_{G}$ ratios in defective graphene are significantly influenced by the doping level of graphene, which may result in a false interpretation of the $\mathrm{I}_{D} / \mathrm{I}_{G}$ ratios $[50]$.

The Raman measurements of the irradiated samples of set A were performed with a Renishaw InVia Raman microscope equipped with a $532 \mathrm{~nm}$ laser for excitation (laser power $2 \mathrm{~mW}$ ). Raman maps were taken in two areas, where graphene was suspended and supported on the $\mathrm{SiO}_{2}$ substrate. A typical measurement is shown in Fig. 1 (b). To obtain the $\mathrm{A}_{D} / \mathrm{A}_{G}$ data for suspended graphene, a Raman map was recorded with the step width of $500 \mathrm{~nm}$ which allows us to average the $\mathrm{A}_{D} / \mathrm{A}_{G}$ data over about 30-40 point spectra. The results show that the $\mathrm{A}_{D} / \mathrm{A}_{G}$ is significantly higher on the suspended graphene area compared to the $\mathrm{SiO}_{2}$ supported graphene. These results are in agreement with irradiation experiments with field effect transistors based on supported graphene [51], where it was observed that highly charged ions (HCIs) yield more damage than SHIs. The SHIs, unlike HCIs, deposit energy deep into the bulk. As a consequence, SHIs heat the substrate efficiently, which may facilitate the healing effect in graphene. The lower damage observed in our experiment for the supported graphene (compared to the suspended) could be explained by such a substrate-assisted mechanism.

The Raman spectra of the samples of set B were measured using an NTMDT NTEGRA Spectra AFM/Raman instrument with $488 \mathrm{~nm}$ excitation wavelength. The output laser power was ca. $4 \mathrm{~mW}$ over a focused spot with a diameter of ca. $0.5 \mu \mathrm{m}$. Several spectra were recorded for each sample at both irradiated and non-irradiated areas (covered by a mask during irradiation) in order to subtract the background signal from the irradiation-induced increase in the $\mathrm{A}_{D} / \mathrm{A}_{G}$ ratio. 


\section{Theoretical approach}

\subsection{Two temperature molecular dynamics model}

We study the SHI-induced damage formation in suspended graphene using two teperature molecular dynamics (TTMD) simulations. This approach is a further development of the classical inelastic thermal spike two temperature model $[52,53]$. The dynamic energy exchange between electronic and ionic subsystems in graphene is followed by using a concurrent multiscale model implemented within a molecular dynamics code [42]. The model assumes that the high velocity electrons generated along the ion path spread out in the target, depositing energy by electronic collisions and exciting electrons. The electronic subsystem becomes eventually thermalized, leading to high local electronic temperatures, and both electronic and lattice subsystem exchange energy with time. In the TTMD model, the evolution of the electronic temperature $\left(T_{e}\right)$ is given by the heat diffusion equation [20] with an additional electron-phonon coupling term $G\left(T_{e}-T_{i}\right)$

$$
C_{e} \frac{\partial T_{e}}{\partial t}=\frac{1}{r} \frac{\partial}{\partial r}\left[r K_{e}\left(T_{e}\right) \frac{\partial T_{e}}{\partial r}\right]-G\left(T_{e}-T_{i}\right)+A(r, t),
$$

where $C_{e}$ is the electronic specific heat capacity, $T_{e}$ and $T_{i}$ are the electronic and ionic temperatures, $r$ is the radial distance from the ion trajectory, $K_{e}$ the electronic thermal conductivity and $t$ is the time. $A(r, t)$ is the energy deposited by secondary electrons as described by the Waligorski radial energy distribution [54]. The evolution of the ionic temperature is naturally followed within the MD algorithm [55] coupled to the Eq.1 by modifying the equations of motion as follows [56]

$$
m_{i} \frac{d^{2} \mathbf{r}_{i}}{d t^{2}}=\mathbf{F}_{i}+\sigma m_{i} \mathbf{v}_{i}
$$

with

$$
\sigma=\frac{G V_{N}\left(T_{e}-T_{i}\right)}{\sum_{i} m_{i}\left(\mathbf{v}_{i}\right)^{2}}
$$

where $m_{i}$ and $\mathbf{r}_{i}$ are the mass and position of atom $i, \mathbf{F}_{i}$ the force acting on it, $\mathbf{v}_{i}$ its velocity. The electronic subsystem is divided in a grid, $V_{N}$ is the volume of an individual electronic grid cell and $T_{e}$ is the electronic 
temperature of the cell. The energy is instantaneously deposited to the electronic subsystem of the target at the beginning of the simulation.

The MD simulations were performed with the PARCAS MD code [57] modified to include the electronic energy exchange [42]. The choice of the interatomic potential in this case is particularly important since the lattice heat capacity and thermal conductivity are intrinsic properties of the potential. In our simulations for the interactions between the carbon atoms, we used the bond-order potential by Brenner et. al. [58] with a longer cutoff $r_{c}=2.64 \AA$ in order to reproduce the bond breaking force more accurately [59]. For small interatomic distances it was smoothly joined to a universal repulsive potential [60] to account for energetic collisions. The chosen carbon potential describes well graphene properties, although, it somewhat underestimates the lattice thermal conductivity of graphene monolayers at room temperature, giving $1100 \mathrm{~W} \mathrm{~K}^{-1} \mathrm{~m}^{-1}$ [61], in contrast to the experimental values, which are in the range 2.000-5.000 $\mathrm{W} \mathrm{K}^{-1} \mathrm{~m}^{-1}[62,63]$. However, we note that these equilibrium values of the lattice thermal conductivity are not directly relevant for energy dissipation during swift heavy ion impacts. This is because the conventional lattice conductivity is to a large extent due to equilibrium phonon properties [64]. In the case of SHI impacts, the ion passage through a graphene monolayer occurs on a sub-fs time scale, and the initial energy dissipation is in the form of a shock wave that propagates out from the impact point on a sub-ps time scale. Since the phonon relaxation time in graphene is larger than a ps [65], it is clear that there is not sufficient time to form an equilibrium phonon system before most of the energy has already dissipated away. Hence the initial energy dissipation in the form of a shock wave can be expected to be described rather by the speed of sound, which is well described by the Brenner potential for graphene [61]

The simulation cell size was selected after performing a finite size test, the biggest cell used in the test had dimensions $100 \mathrm{~nm} \times 86.4 \mathrm{~nm} \times 7.0 \mathrm{~nm}$. The system used in the simulations consisted of a $25.1 \mathrm{~nm} \times 21.6 \mathrm{~nm} \times 7.0 \mathrm{~nm}$ graphene sheet of 20000 atoms with periodic boundary conditions.

\subsection{Parameterization of the electronic subsystem model.}

The electronic subsystem in our simulations is described by means of the electronic stopping power of a projectile $S_{e}$ and three additional parameters, electron-phonon coupling $G\left(T_{e}\right)$, electronic specific thermal conductivity $K_{e}\left(T_{e}\right)$ and electronic specific heat capacity $C_{e}\left(T_{e}\right)$, which are included in Eq. 1. These parameters can have a strong dependence on the electronic 
temperature, therefore, it is important to consider them as functions of $T_{e}$ in the calculation in order to obtain an accurate description of the temperature evolution.

It is challenging to obtain the electronic stopping power of graphene directly from experiments. Instead we estimate $S_{e}$ for graphene from the value given by SRIM [66] for equilibrium charge state ions in graphite. We scale this value to match the energy deposited to a single sheet of graphene in graphite dividing the stopping power given in $\mathrm{keV} / \mathrm{nm}$ by three, since there are approximately 3 monolayers per nm in graphite. The stopping power from SRIM should be a good approximation for most of the ions. However, for $23 \mathrm{MeV} \mathrm{I}$ and $35 \mathrm{MeV} \mathrm{Au}$ ions the experimental charge states are considerably lower than the equilibrium values, and therefore we expect SRIM to overestimate the stopping power of these two ions.

The $S_{e}$ values for the ions used in our experiments vary from $0.27 \mathrm{keV} /$ layer to $5.00 \mathrm{keV} /$ layer (see table 1). Five different simulations were carried out for each ion in order to account for stochastic uncertainties.

Table 1: The studied ions and energies with the corresponding stopping power values calculated from the values of graphite (given by SRIM) for graphene, as well as the corresponding electronic stopping cross-sections for graphite. The superscripts denote the charge state of the ions used in our experiments.

\begin{tabular}{lccccc}
\hline ion & sample & $\begin{array}{c}\mathrm{E} \\
{[\mathrm{MeV}]}\end{array}$ & $\begin{array}{c}S_{e} \\
{[\mathrm{keV} / \text { layer }]}\end{array}$ & $\begin{array}{c}S_{e} \\
{[\mathrm{eVnm} / \text { atom }]}\end{array}$ & $\begin{array}{c}S_{n} \\
{[\mathrm{eV} / \text { layer }]}\end{array}$ \\
\hline $\mathrm{C}^{6+}$ & $\mathrm{B}$ & 35 & 0.27 & 7.10 & 0.2 \\
$\mathrm{C}^{4+}$ & $\mathrm{B}$ & 23 & 0.35 & 9.21 & 0.2 \\
$\mathrm{O}^{1+}$ & $\mathrm{A}$ & 1 & 0.44 & 11.56 & 7.0 \\
$\mathrm{O}^{2+}$ & $\mathrm{A}$ & 3 & 0.72 & 18.94 & 3.1 \\
$\mathrm{Si}^{3+}$ & $\mathrm{A}$ & 6 & 1.22 & 32.10 & 7.3 \\
$\mathrm{Si}^{6+}$ & $\mathrm{B}$ & 35 & 1.37 & 36.05 & 1.7 \\
$\mathrm{Si}^{4+\text { and }} 3+$ & $\mathrm{A}, \mathrm{B}$ & 15 & 1.48 & 38.94 & 3.4 \\
$\mathrm{I}^{6+}$ & $\mathrm{A}$ & 23 & 2.20 & 57.89 & 91.3 \\
$\mathrm{Au}^{7+}$ & $\mathrm{B}$ & 35 & 2.24 & 58.95 & 183.3 \\
$\mathrm{Ta}^{24+}$ & $\mathrm{A}$ & 84 & 4.90 & 128.93 & 78.1 \\
$\mathrm{Xe}^{23+}$ & $\mathrm{A}$ & 91 & 5.00 & 131.56 & 33.3 \\
\hline
\end{tabular}

The electron-phonon coupling $G\left(T_{e}\right)$ is estimated considering the relation between the relaxation time $\tau$ and the electronic specific heat capacity 
$C_{e}\left(T_{e}\right), \tau=C_{e}\left(T_{e}\right) / G\left(T_{e}\right)$ [67]. The constant $\tau$ can be obtained using timeand angle-resolved photoemission spectroscopy. If homogeneous heating is applied to the electron subsystem, and after it is allowed to relax, the electronic temperature drops according to $\exp (-t / \tau)$, where $t$ is the time, and therefore the constant $\tau$ can be measured. For graphene, the characteristic relaxation time due to the interaction with optical phonons is $\tau \approx 150 \mathrm{fs}$ [68]. In our simulations $G\left(T_{e}\right)$ takes values from $1 \cdot 10^{15} \mathrm{Wm}^{-3} \mathrm{~K}^{-1}$ at $300 \mathrm{~K}$ to $2 \cdot 10^{18} \mathrm{Wm}^{-3} \mathrm{~K}^{-1}$ at the highest temperatures.

The heat capacity, $C_{e}\left(T_{e}\right)$, was evaluated numerically through

$$
C_{e}\left(T_{e}\right)=\frac{\partial}{\partial T_{e}} \int_{-\infty}^{\infty} g(E) f\left(E, \mu\left(T_{e}\right), T_{e}\right) E d E,
$$

where the $f\left(E, \mu\left(T_{e}\right), T_{e}\right)$ is the Fermi-Dirac distribution, and the dependence of the electron chemical potential $\mu_{e}$ on $T_{e}$ was taken into account by solving numerically the integral equation connecting the total number of charge carriers with $\mu_{e}$. We stress that the position of the Fermi energy at high temperatures is considerably different from $E_{F, 0}=\mu\left(T_{e}=0\right)$. The density of states(DOS) $g(E)$ was calculated using a standard density functional-theory approach with the PBE exchange and correlation functional, as implemented in the VASP code[69]. Since in SHI irradiation electrons reach temperatures over $50.000 \mathrm{~K}$, we need an accurate description of the high energy states of these DOS. As the contribution of the states above the vacuum level (note that graphene ionization potential is about $5 \mathrm{eV}$ in our model) is rather small even at the highest temperatures studied, for the calculation of $C_{e}$ we used the density of states of A-A stacked graphite, thus avoiding the unphysical contribution of the vacuum states at high energies, which depends on the simulation box size.

As graphene is frequently doped, we choose three values of $E_{F}=0.01 \mathrm{eV}$, $E_{F}=0.20 \mathrm{eV}$ and $E_{F}=0.40 \mathrm{eV}$, corresponding to charge carrier (electron) concentrations of $7 \times 10^{9}, 3 \times 10^{12}$, and $13 \times 10^{12} \mathrm{~cm}^{-2}$, respectively. $C_{e}\left(T_{e}\right)$ is presented in Fig 2, and it is similar to $C_{e}\left(T_{e}\right)$ calculated using a model density of states in the low-temperature range [68]. Evidently, at low temperatures $C_{e}\left(T_{e}\right)$ strongly depends on charge carrier concentration, and $C_{e}$ is higher for larger $n$, as expected, while at high $T_{e}$ it is essentially independent of $E_{F}$.

The electronic thermal conductivity $K_{e}\left(T_{e}\right)$ of graphene is believed to account only for $1 \%$ of the total thermal conductivity [70] at temperatures close and slightly above room temperature. Yigen et al. [71] reported electron 


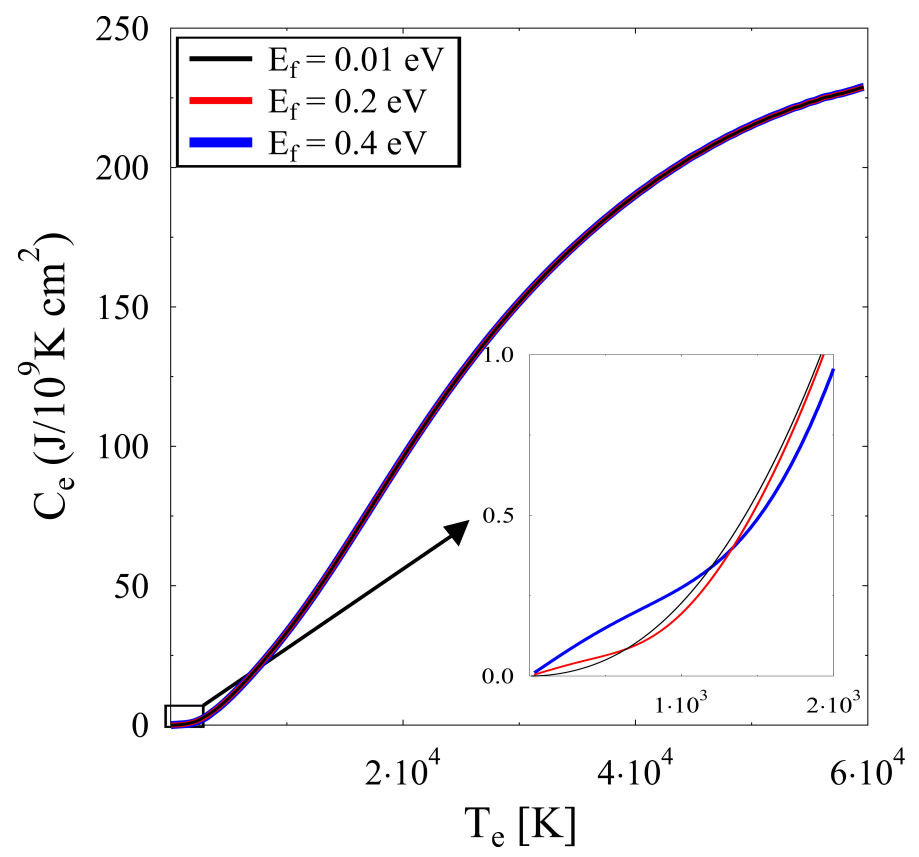

Figure 2: (a) Electronic temperature dependence of the specific electronic heat capacity $C_{e}\left(T_{e}\right)$ of A-A stacked graphite for three different chemical potentials $E_{F}=0.01 \mathrm{eV}$, $E_{F}=0.20 \mathrm{eV}$ and $E_{F}=0.4 \mathrm{eV}$.

thermal conductivity values ranging from $0.5-10 \mathrm{Wm}^{-1} \mathrm{~K}^{-1}$ for the temperature interval 20-300 K. The most crucial transfer of energy in SHI irradiation happens in the first picoseconds in the region around the ion track, where the electrons reach temperatures of several tens of thousands of K. At high temperatures under the Fermi temperature $T_{F} \sim 20.000 \mathrm{~K}$, we expect the electron-electron scattering to dominate. In that regime, the scattering lifetime follows $\frac{1}{\tau_{e-e}} \propto T^{2} \ln T[72,73]$ and therefore the electronic thermal conductivity decreases $K_{e}=\frac{C v^{2} \tau}{2}$. At temperatures higher than $T_{F}$ the electron subsystem starts to behave like a low density plasma and $K_{e}$ rises again [56].

It is reasonable to assume that $K_{e}$ depends only weakly on the doping level at high temperatures, since the electronic heat capacity converges to the same value independently of the Fermi energy. Due to the fact that there is no experimental data of the thermal conductivity at the temperatures of interest, we leave $K_{e}$ as a free parameter, and we fit it to obtain a reasonable agreement with the threshold for damage formation that is observed in the experiments. 


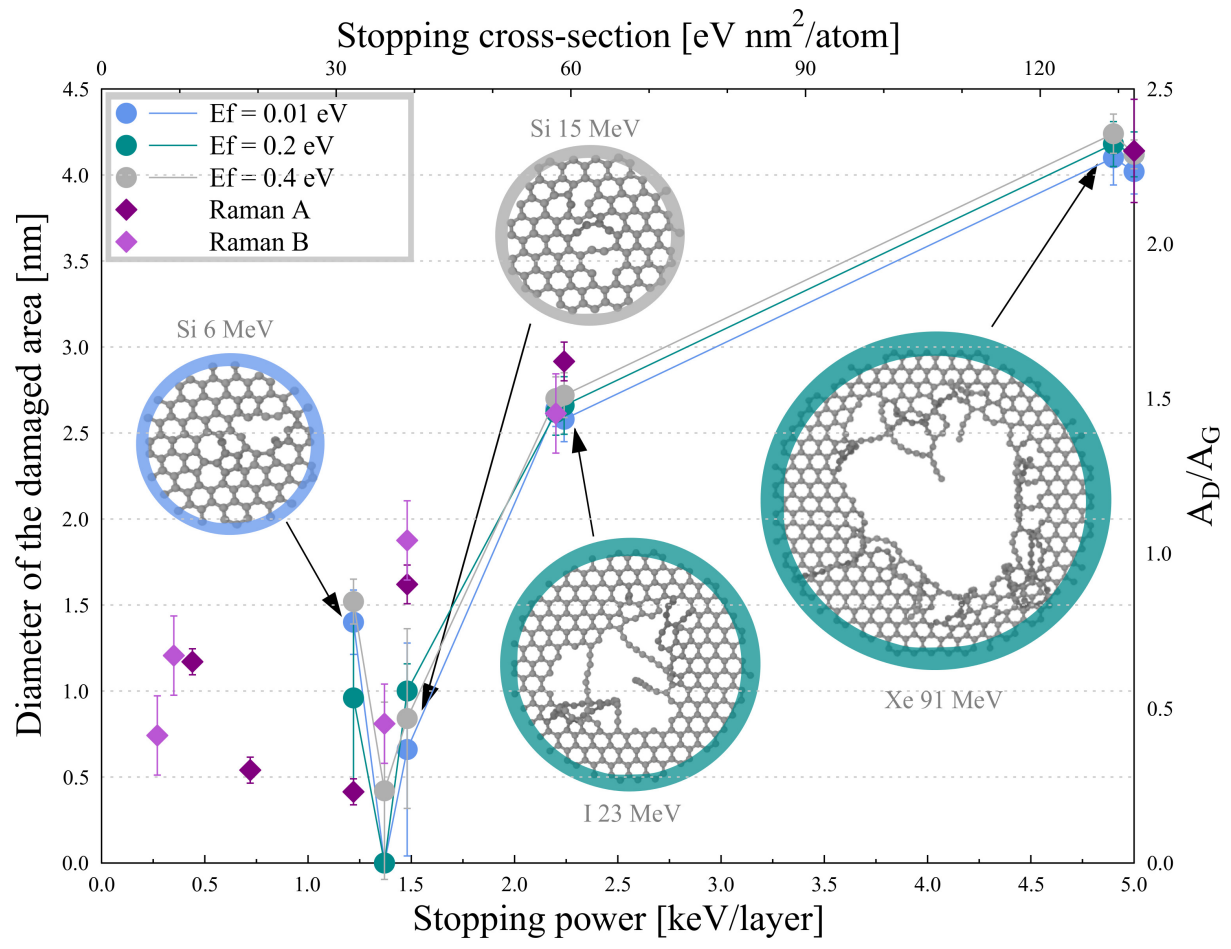

Figure 3: Defect size as a function of the energy deposited in the graphene layer. Simulation results with the average defect diameter correspond to the $y$-axis on left side of the figure, and the Raman results with the $D$ and $G$ mode area ratio for the samples $\mathrm{A}$ and $\mathrm{B}$ correspond to the axis on the right side. Simulations are presented with three different chemical potentials corresponding to energies $E_{F}=0.01 \mathrm{eV}, E_{F}=0.20 \mathrm{eV}$ and $E_{F}=0.4 \mathrm{eV}$. Snapshots from simulations corresponding to three different stopping power values are indicated.

Since it is a fitted parameter, we ignore currently its dependence on the electronic temperature and therefore use it as a constant value.

\section{Results and discussion}

Raman spectra from the graphene samples of set A irradiated with various ions with different stopping powers $S_{e}$ are shown in Fig. 1 (c). The corresponding $\mathrm{A}_{D} / \mathrm{A}_{G}$ ratios are plotted in Fig. 1 (d) against $S_{e}$ (as calculated with SRIM). For ions with low $S_{e}$ values up to $1.22 \mathrm{keV} /$ layer, the $\mathrm{A}_{D} / \mathrm{A}_{G}$ ratio decreases with increasing stopping power. From $1.22 \mathrm{keV} /$ layer 
onwards, the $\mathrm{A}_{D} / \mathrm{A}_{G}$ ratio clearly increases with increasing electronic stopping power. The latter can easily be explained in terms of increasing a defect (pore) size.

The origin of the decrease for low electronic stopping powers seems, however, less clear. There are several possible explanations, the contribution of which is difficult to disentangle. For instance, the charge state of the projectile may play a certain role in this process, since not all projectiles used in the study had their equilibrium charge state. Moreover, the morphology of the defect (hollow areas vs. defective areas, round pores vs elongated pores) must also be taken into account, especially for these rather small-size defects. The presence of water, on the other hand, may introduce the chemistry related effects at the reactive edges [74], which may influence the Raman data. Finally, synergistic effects of nuclear and electronic stopping(see ref [75]) cannot be excluded in this region. To clarify which of the above-listed factors may play a crucial role for the reverse behavior of the damage dependence on the stopping power, a dedicated separate study must be performed. Above $1.22 \mathrm{keV} /$ layer, the energy transferred to the target in a collision is more than the threshold displacement energy of the lattice atoms (ca. $23 \mathrm{eV}[76]$ ) and pore formation becomes more and more effective. Thus, $\mathrm{A}_{D} / \mathrm{A}_{G}$ starts to increase, mirroring the increasing defect size.

Note also that the nuclear stopping power $S_{n}$ of the projectiles used in our study (see table 1) is in general very small, of the order of a few $10 \mathrm{eV}$ per atomic layer. If the energy transferred in a collision is smaller than the threshold displacement energy of $23 \mathrm{eV}, S_{n}$ might cause wobbling of the graphene membrane without creating a defect. This could also give rise to purification effects, causing desorption of loosely bound contamination atoms from the graphene sheet. Although the energy deposited by nuclear stopping is somewhat higher for $\mathrm{Si}$ ions of $6 \mathrm{MeV}$ (third value in Fig. 1 (d)) than that for $\mathrm{O}$ ions of 1 and $3 \mathrm{MeV}$ (first and second values in Fig. 1(d)), all these values are well below the displacement threshold energy, allowing us to safely exclude nuclear stopping as the reason for decreasing of $\mathrm{A}_{D} / \mathrm{A}_{G}$ ratio in the lowest energy range studied here.

In Figure 3 we compare the simulation results with the Raman measurements. The Raman experiments indicate a threshold for damage production between $S_{e}$ values of $1.22 \mathrm{keV} /$ layer and $1.48 \mathrm{keV} /$ layer. We see a clear correlation within the statistical uncertainty between the experimental $\mathrm{A}_{D} / \mathrm{A}_{G}$ ratio and the simulation results of the defect size, obtained using electronic thermal conductivity $K_{e} 1 \mathrm{Wm}^{-1} \mathrm{~K}^{-1}$. The linear correlation suggests that 
the defects are pore-like, since for that type of defects the Raman active area and the $\mathrm{A}_{D} / \mathrm{A}_{G}$ ratio scale linearly with the diameter of the pore. Despite the good agreement, we note that in the simulations the trend of the damage produced by Si ions with the energies $6 \mathrm{MeV}, 15 \mathrm{MeV}$ and $35 \mathrm{MeV}$ differs from the $\mathrm{A}_{D} / \mathrm{A}_{G}$ curve given by the Raman experiments. In our simulations we observe smaller damaged area by the $\mathrm{Si}(15 \mathrm{MeV})$ and $\mathrm{Si}(35$ $\mathrm{MeV})$ ions than by the $\mathrm{Si}(6 \mathrm{MeV})$ ions, even though the former two have higher stopping powers. The reason for this behaviour lies in the Waligorski energy distribution formula [54], which gives the radial distribution of the secondary electrons around the ion track. According to the Waligorski formula, higher velocity ions will transfer on average more momentum to the secondary electrons and therefore these will travel further. Hence if two ions have approximately the same stopping power but one of them has higher velocity, the energy deposition profile for the latter ion will be less localized at the centre of the track and on average the electron temperatures will be lower than for the ion with lower velocity. This effect was seen in the bulk materials [77] and is known as the velocity effect. The velocity effect also explains why we barely observe any damage by the $\mathrm{Si}(35 \mathrm{MeV})$ ions contradictory to the experimental results. However, the experiments are in good agreement with the work of Zeng et al. [32] where is reported that supported graphene shows no velocity effect, unlike graphite. These results indicate the necessity of further development of theoretical models to enable a better understanding of the velocity effect in two-dimensional materials.

To analyse the effect of doping on the damage production by SHI, we repeated the simulations with different chemical potentials $\left(E_{F}=0.01 \mathrm{eV}\right.$, $E_{F}=0.20 \mathrm{eV}$ and $E_{F}=0.4 \mathrm{eV}$ ). As one can see in Figure 3, the results showed only small deviation due to the changes in the Fermi energy. The largest differences were observed in the simulations with low stopping powers, which have also large statistical uncertainties. In some simulations with low stopping powers, no damage was observed, increasing considerably the standard deviation of the data. Experimental and simulation results are both clearly lower than the value reported in Ref. [16] for the damage formation threshold, underlining the importance of the temperature dependence of the parameters of the electronic subsystem as well as the electron-phonon coupling during the SHI impact.

We chose the case of $84 \mathrm{MeV}$ Ta irradiation $\left(S_{e} 4.9 \mathrm{keV} /\right.$ layer $)$ to demonstrate more closely the evolution of pore formation in the simulations. The snapshots of the atomic structure at different times are shown in Fig 4. Right 


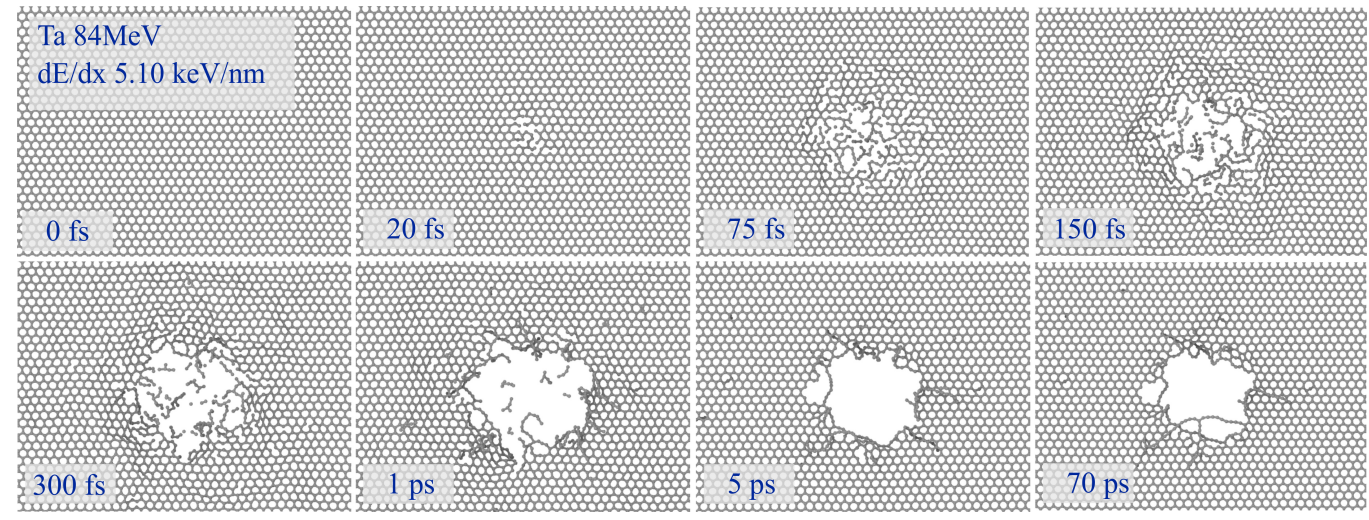

Figure 4: Visualization of pore formation in the simulations. Top view of graphene is presented after irradiation with $84 \mathrm{MeV}$ Ta corresponding to a stopping power of 4.9 $\mathrm{keV} /$ layer. The final pore diameter is about $41 \AA$.

after the initial energy deposition, defects start to appear in the sample and a cylindrical area of amorphous material is created at the path of the ion within the first femtoseconds of the simulation. At $20 \mathrm{fs}$, the kinetic energy transferred from the electronic subsystem to the atoms is sufficient to displace the atoms from their initial lattice sites within a diameter of about $15 \AA$. The energy spreads fast and already at 75 fs the defected area has spread to a diameter of about $45 \AA$. After the initial defect has formed, graphene reknits or self-organizes [78] itself at the edges, and as a result after about $500 \mathrm{fs}$ the final defect diameter of about $41 \AA$ is reached and a round pore is created. This pore corresponds to about 460 missing carbon atoms. Atoms detached from the continuous network are either sputtered, re-deposited as adatoms or captured at the sides of the created hole. In addition to the large hole-type defect, vacancies and adatoms can be created during the irradiation. The total simulation time was $70 \mathrm{ps}$, allowing relaxation of the structure after the final defect size had been reached.

As mentioned above, the best agreement with the Raman experimental results was achieved (see Figure 3) when the specific electronic thermal conductivity $K_{e}$ of graphene was estimated to be $1 \mathrm{Wm}^{-1} \mathrm{~K}^{-1}$. We note that this value is well within the range of values reported in the literature [71, 70]. For higher thermal conductivities, $\mathrm{Si}\left(6 \mathrm{MeV}, S_{e}=1.22 \mathrm{keV} /\right.$ layer $)$ and $\mathrm{Si}(15$ $\mathrm{MeV}, S e_{e}=1.48 \mathrm{keV} /$ layer) ions do not produce damage in the graphene layer, and for lower $K_{e}$ we still do not observe any permanent damage with 
lower stopping power ions $\mathrm{O}\left(1 \mathrm{MeV}, S_{e}=0.44 \mathrm{keV} /\right.$ layer $)$ and $\mathrm{O}(3 \mathrm{MeV}$, $S_{e}=0.72 \mathrm{keV} /$ layer) ions.

Higher $K_{e}\left(T_{e}\right)$ rapidly decrease the defect production in graphene, as heat dissipates faster through the electronic subsystem. With the values close to $300 \mathrm{Wm}^{-1} \mathrm{~K}^{-1}$ (the highest reported values in literature [79]) no structural changes in graphene are seen in the simulations for all studied ions.

\section{Conclusions}

In this work we described a method to estimate quantitatively the size of the SHI-induced pore-like defects in suspended graphene by combination of Raman spectroscopy and two temperature molecular dynamics simulations. We showed that the irradiation with SHIs of increasing stopping power produces defects with increasing size in the suspended graphene. According to our simulations, regardless of the high lattice thermal conductivity of graphene, interactions during high energy ion irradiation in the $\mathrm{MeV}$ range are enough to produce pore-type defects of diameter size ranging between 1-4 $\mathrm{nm}$ in the membrane, which is the pore size required for advanced nanofiltration concepts aiming e.g. at the desalination of water. The good agreement between experiments and simulations at high stopping powers suggests that the Raman signal is due to the formation of pores in the layer. Curiously, at low electronic stopping power values Raman mapping shows a healing effect. The defect production threshold for the stopping power is found to be ca. 1.22-1.48 keV/layer. Above this, the defect size sensitively depends on the stopping power, indicating diameter-selectivity for the created pores. The simulations with the concurrent two temperature molecular dynamics model coupling the ionic and electronic subsystems suggest that the electronic thermal conductivity of our graphene samples at high electronic temperatures is rather small. By comparing predictions of the simulations with the Raman experiments, we estimated the electronic thermal conductivity of graphene at high temperatures to be about $1 \mathrm{Wm}^{-1} \mathrm{~K}^{-1}$, which is well in the range of values reported in the literature. The chemical potential proved to have only a minor effect on the defect size. Controllable modification of graphene atomic structure opens a way for patterning suspended samples for application purposes, such as creating detectors with high sensitivity and membranes with unprecendented filtering performance. 


\section{Acknowledgments}

HV thanks MATRENA doctoral programme and Helsinki Institute of Physics for supporting financially this project. HA thanks the funding from Academy of Finland as well as discussions with Szymon Daraszewicz. OO, RK and MS acknowledge funding and fruitful discussions within the priority program SPP 1495 "Graphene" and the collaborative research center SFB 1242 "Non-equilibrium dynamics in condensed matter in the time domain" funded by the Deutsche Forschungsgemeinschaft. MK and MJ acknowledge the Croatian Centre of Excellence for Advanced Materials and Sensing Devices and the Croatian Science Foundation (pr. no. 8127) which supported this investigation. MS, HL, and MK acknowledge support from the DFG through project no. SCHL 384/16-1. AVK further acknowledges financial support from the Academy of Finland through Projects no. 263416 and 286279 and the Ministry of Education and Science of the Russian Federation in the framework of Increase Competitiveness Program of NUST "MISiS" (\#K2-2015-033). We also thank the CSC-IT Center for Science Ltd for generous grants of computer time. JK acknowledges the Wiener Wissenschafts-, Forschungs- und Technologiefonds for founding through project MA14-009.

[1] G. F. Schneider, S. W. Kowalczyk, V. E. Calado, G. Pandraud, H. W. Zandbergen, L. M. K.Vandersypen, C. Dekker, DNA Translocation through Graphene Nanopores, Nano Lett. 10 (2010) 3163-3167.

[2] T. H. Han, Y.-K. Huang, A. T. L. Tan, V. P. Dravid, J. Huang, Steam Etched Porous Graphene Oxide Network for Chemical Sensing, J. Am. Chem. Soc. 133 (2011) 15264-15267.

[3] D. Jiang, V. R. Cooper, S. Dai, Porous Graphene as the Ultimate Membrane for Gas Separation, Nano Lett. 9 (2009) 4019-4024.

[4] J. Schrier, Helium Separation Using Porous Graphene Membranes, J. Phys. Chem. Lett. 1 (2010) 2284-2287.

[5] S. Koenig, L. Wang, J. Pellegrino, J. S. Bunch, Selective molecular sieving through porous graphene, Nature Nanotech. 7 (2012) 728-732.

[6] D. Cohen-Tanugi, J. C. Grossman, Water Desalination across Nanoporous Graphene, Nano Lett. 12 (2012) 3602-3608. 
[7] U. Bangert, A. Bleloch, M. H. Gass, A. Seepujak, J. van den Berg, Doping of few-layered graphene and carbon nanotubes using ion implantation, Phys. Rev. B 81 (2010) 245423.

[8] U.Bangert, W. Pierce, D. M. Kepaptsoglou, Q. Ramasse, R. Zan, M. H. Gass, J. A. V. den Berg, C. B. Boothroyd, J. Amani, H. Hohsäss, Ion Implantation of Graphene - Toward IC Compatible Technologies, Nano Lett. 13 (2013) 4902-4907.

[9] E. H. Åhlgren, J. Kotakoski, A. V. Krasheninnikov, Atomistic simulations of the implantation of low-energy boron and nitrogen ions into graphene, Phys. Rev. B 83 (2011) 115424.

[10] E. H. Åhlgren, J. Kotakoski, O. Lehtinen, A. V. Krasheninnikov, Ion irradiation tolerance of graphene as studied by atomistic simulations, App. Phys. Lett. 23 (2012) 233108.

[11] C.-T. Pan, J. A. Hinks, Q. M. Ramasse, G. Greaves, U. Bangert, S. E. Donnelly, S. J. Haigh, In-situ observation and atomic resolution imaging of the ion irradiation induced amorphisation of graphene, Sci. Rep. 4 (2014) 6334. doi:10.1038/srep06334.

[12] O. Lehtinen, J. Kotakoski, A. V. Krasheninnikov, J. Keinonen, Cutting and controlled modification of graphene with ion beams, Nanotechnol. 22 (2011) 175306.

[13] J. Kotakoski, C. Brand, Y. Lilach, O. Cheshnovsky, C. Mangler, M. Arndt, J. C. Meyer, Toward two-dimensional all-carbon heterostructures via ion beam patterning of single-layer graphene, Nano Lett.doi: 10.1021/acs. nanolett.5b02063.

[14] M. C. Lemme, D. C. Bell, J. R. Williams, L. A. Stern, B. W. H. Baugher, P. Jarillo-Herrero, C. M. Marcus, Etching of graphene devices with a helium ion beam, ACS Nano 3 (9) (2009) 2674-2676.

[15] D. C. Bell, M. C. Lemme, L. a. Stern, J. R. Williams, C. M. Marcus, Precision cutting and patterning of graphene with helium ions., Nanotechnology 20 (2009) 455301. 
[16] S. Zhao, J. Xue, Modification of graphene supported on sio 2 substrate with swift heavy ions from atomistic simulation point, Carbon 93 (2015) 169.

[17] M. Caron, H. Rothard, M. Toulemonde, B. Gervais, M. Beuve, Theoretical and experimental study of electronic temperatures in heavy ion tracks from Auger electron spectra and thermal spike calculations, Nucl. Instrum. Methods Phys. Res. B 245 (2006) 36.

[18] R. L. Fleischer, P. B. Price, R. M. Walker, Ion explosion Spike Mechanism for Formation of Charged-Particle Tracks in Solids, J. Appl. Phys. 36 (1965) 3645.

[19] Y. Cherednikov, S. N. Sun, H. M. Urbassek, Hybrid particle-incell/molecular dynamics simulation of swift-ion tracks in LiF, Phys. Rev. B 87 (2013) 245424.

[20] G. Szenes, Comparison of two thermal spike models for ion-solid interaction, Nucl. Instr. Meth. Phys. Res. B 269 (2011) 174-179.

[21] C. Trautmann, S. Klaumünzer, H. Trinkaus, Effect of Stress on Track Formation in Amorphous Iron Boron Alloy: Ion Tracks as Elastic Inclusions, Phys. Rev. Lett. 85 (2000) 3648.

[22] N. Itoh, A. M. Stoneham, Excitonic model of track registration of energetic heavy ions in insulators, Nucl. Instr. Meth. Phys. Res. B 146 (1998) 362-366.

[23] M. C. Ridgway, T. Bierschenk, R. Giulian, B. Afra, M. D. Rodriguez, L. L. Araujo, A. P. Byrne, N. Kirby, O. H. Pakarinen, F. Djurabekova, K. Nordlund, M. Schleberger, O. Osmani, N. Medvedev, B. Rethfeld, P. Kluth, Tracks and Voids in Amorphous Ge Induced by Swift HeavyIon Irradiation, Phys. Rev. Lett. 110 (2013) 245502.

[24] F. Agulló-López, A. Mendez, G. García, J. Olivares, J. M. Cabrera, Synergy between thermal spike and exciton decay mechanisms for ion damage and amorphization by electronic excitation, Phys. Rev. B 74 (2006) 174109. doi:10.1103/PhysRevB.74.174109.

URL http://link.aps.org/doi/10.1103/PhysRevB.74.174109 
[25] D. Kanjijal, Swift heavy ion-induced modification and track formation in materials, Current Science 80 (12) (2001) 1560.

[26] S. Akcöltekin, H. Bukowska, T. Peters, O. Osmani, I. Monnet, I. Alzaher, B. B. d'Etat, H. Lebius, M. Schleberger, Unzipping and folding of graphene by swift heavy ions, Appl. Phys. Lett. 98 (2011) 103103.

[27] O. Ochedowski, S. Akcöltekin, B. Ban-d'Etat, H. Lebius, M. Schleberger, Detecting swift heavy ion irradiation effects with graphene, Nucl. Inst. and Meth. in Phys. Res. B 314 (2013) 18-20.

[28] S. Kumar, A. Tripathi1, F. Singh, S. A. Khan, V. Baranwal, D. K. Avasthi, Purification/annealing of graphene with $100-\mathrm{MeV}$ Ag ion irradiation, Nanoscale Res Lett. 9 (2014) 126.

[29] O. Ochedowski, K. Marinov, G. Wilbs, G. Keller, N. Scheuschner, D. Severin, M. Bender, J. Maultzsch, F. J. Tegude, M. Schleberger, Radiation hardness of graphene and $\mathrm{MoS}_{2}$ field effect devices against swift heavy ion irradiation, Nanoscale Res Lett. 113 (2013) 214306.

[30] S. Kumar, A. Tripathi, S. A. Khan, C. Pannu, D. K. Avasthi, Radiation stability of graphene under extreme conditions, Appl. Phys. Lett. 105 (2014) 133107.

[31] O. Ochedowski, B. K. Bussmann, B. B. d'Etat, H. Lebius, M. Schleberger, Manipulation of the graphene surface potential by ion irradiation, Appl. Phys. Lett. 102 (2013) 153103.

[32] J. Zeng, J. Liu, H. Yao, P. Zhai, S. Zhang, H. Guo, P. Hu, J. Duan, D. Mo, M. Hou, Y. Sun, Comparative study of irradiation effects in graphite and graphene induced by swift heavy ions and highly charged ions, Carbon 100 (2016) 16-26. doi:10.1016/j.carbon.2015.12.101. URL http://www.sciencedirect.com/science/article/pii/ S0008622315305674

[33] O. Ochedowski, O. Lehtinen, U. Kaiser, A. Turchanin, B. Ban-d'Etat, H. Lebius, M. Karlušić, M. Jakšić, M. Schleberger, Nanostructuring graphene by dense electronic excitation, Nanotechnology 26 (46) (2015) 465302.

URL http://stacks.iop.org/0957-4484/26/i=46/a=465302 
[34] P. Kluth, C. S. Schnohr, O. H. Pakarinen, F. Djurabekova, D. J. Sprouster, R. Giulian, M. C. Ridgway, A. P. Byrne, C. Trautmann, D. J. Cookson, K. Nordlund, M. Toulemonde, Fine structure in swift heavy ion tracks in amorphous $\mathrm{sio}_{2}$, Physical Review Letters 101 (2008) 175503, also availalbe at http://dx.doi.org/10.1103/PhysRevLett. 101.175503. doi:10.1103/PhysRevLett.101.175503.

[35] A. Debelle, M. Backman, L. Thomé, W. J. Weber, M. Toulemonde, S. Mylonas, A. Boulle, O. H. Pakarinen, N. Juslin, F. Djurabekova, K. Nordlund, F. Garrido, Combined experimental and computational study of the recrystallization process induced by electronic interactions of swift heavy ions with silicon carbide crystals, Physical Review B 86 (2012) 100102(R), also available at http://dx.doi.org/10.1103/ PhysRevB.86.100102. doi:10.1103/PhysRevB.86.100102.

[36] O. H. Pakarinen, F. Djurabekova, K. Nordlund, Density evolution in formation of swift heavy ion tracks in insulators, Nuclear Instruments and Methods in Physics Research B 268 (2010) 3163, available at http: //dx.doi.org/10.1016/j.nimb.2010.05.079. doi:10.1016/j.nimb. 2010.05.079.

[37] T. Bierschenk, R. Giulian, B. Afra, M. D. Rodriguez, D. Schauries, S. Mudie, O. H. Pakarinen, F. Djurabekova, K. Nordlund, B. Rethfeld, M. C. Ridgway, P. Kluth, Latent ion tracks in amorphous silicon, Phys. Rev. B 88 (2013) 174111.

[38] M. Ridgway, T. Bierschenk, R. Giulian, B. Afra, M. D. Rodriguez, L. Araujo, A. P. Byrne, N. Kirby, O. H. Pakarinen, F. Djurabekova, K. Nordlund, M. Schleberger, O. Osmani, N. Medvedev, B. Rethfeld, W. Wesch, P. Kluth, Track and voids in amorphous ge induced by swift heavy-ion irradiation, Phys. Rev. Lett. 110 (2013) 245502.

[39] A. A. Leino, O. H. Pakarinen, F. Djurabekova, K. Nordlund, P. Kluth, M. C. Ridgway, Swift heavy ion induced shape transformation of au nanocrystals mediated by molten material recrystallization, Materials Research Letters 2 (1) (2014) 37-42. doi:10.1080/21663831.2013.856816.

URL http://www.tandfonline.com/doi/pdf/10.1080/21663831. 2013.856816 
[40] P. A. F. P. Moreira, R. Devanathan, W. J. Weber", Atomistic simulation of track formation by energetic recoils in zircon, J. Phys.: Condens. Matter 22 (2010) 395008.

[41] D. Schwen, E. M. Bringa, Atomistic simulations of swift ion tracks in diamond and graphite, Nucl. Instr. Meth. Phys. Res. B 256 (2007) 187192.

[42] A. A. Leino, S. L. Daraszewicz, O. H. Pakarinen, K. Nordlund, F. Djurabekova, Atomistic two-temperature modelling of ion track formation in silicon dioxide, Europhysics Letters 110 (1) (2015) 16004, also available at http://stacks. iop.org/0295-5075/110/i=1/a=16004.

[43] B. Afra, M. D. Rodriguez, C. Trautmann, O. H. Pakarinen, F. Djurabekova, K.Nordlund, T. Bierschenk, R. Giulian, M. C. Ridgway, G. Rizza, N. Kirby, M. Toulemonde, P. Kluth, Saxs investigations of the morphology of swift heavy ion tracks in $\alpha$-quartz, Journal of Physics: Condensed matter 25 (2013) 0455006, also available at stacks.iop.org/JPhysCM/25/045006.

[44] A. A. Leino, S. L. Daraszewicz, O. H. Pakarinen, F. Djurabekova, K. Nordlund, B. Afra, P. Kluth, Structural analysis of simulated swift heavy ion tracks in quartz, Nuclear Instruments and Methods in Physics Research B 326 (2014) 289 - 292, also available at http://dx.doi.org/ 10.1016/j.nimb.2013.10.075. doi:10.1016/j.nimb.2013.10.075.

[45] A. C. Ferrari, J. C. Meyer, V. Scardaci, C. Casiraghi, M. Lazzeri, F. Mauri, S. Piscanec, D. Jiang, K. S. Novoselov, S. Roth, A. K. Geim, Raman Spectrum of Graphene and Graphene Layers, Phys. Rev. Lett. 97 (2006) 187401.

[46] A. Reina, X. Jia, J. Ho, D. Nezich, H. Son, V. Bulovic, M. S. Dresselhaus, J. Kong, Large area, few-layer graphene films on arbitrary substrates by chemical vapor deposition, Nano Letters 9 (1) (2009) 30-35, pMID: 19046078. arXiv:http://dx.doi.org/10.1021/nl801827v, doi:10. 1021/n1801827v.

URL http://dx.doi.org/10.1021/n1801827v

[47] A. C. Ferrari, J. Robertson, Interpretation of Raman spectra of disordered and amorphous carbon, Phys. Rev. B 61 (2000) 14095. 
[48] C. Thomsen, S. Reich, Double Resonant Raman Scattering in Graphite, Phys. Rev. Lett. 85 (2000) 5214.

[49] M. Lucchesea, F. Stavalea, E. M. Ferreiraa, C. Vilania, M. Moutinhob, R. B. Capaza, C. Achetea, A. Jorio, Quantifying ion-induced defects and Raman relaxation length in graphene, Carbon 48 (2010) 1592-1597.

[50] M. Bruna, A. K. Ott, M. Ijäs, D. Yoon, U. Sassi, A. C. Ferrari, Doping Dependence of the Raman Spectrum of Defected Graphene, ACS Nano 8 (2014) 7432-7441.

[51] P. Ernst, R. Kozubek, L. Madauß, J. Sonntag, A. Lorke, M. Schleberger, Irradiation of graphene field effect transistors with highly charged ions, Nuclear Instruments and Methods in Physics Research Section B: Beam Interactions with Materials and Atoms (2016) doi:http://dx.doi.org/10.1016/j.nimb.2016.03.043.

URL http://www.sciencedirect.com/science/article/pii/ S0168583X16300246

[52] A. Meftah, F. Brisard, J. Costantini, E. Dooryhee, M. Hage-Ali, M. Hervieu, J. Stoquert, F. Studer, M. Toulemonde, Track formation in sioz quartz and the thermal-spike mechanism, Physical Review B.

[53] Z. Wangts, C. Dufourtt, E. Paumiertt, M. Toulemoude, The se sensitivity of metals under swift-heavy-ion irradiation a transient thermal process, Journal of Physics: Condens. Matter 6 (1994) 6733 - 6750.

[54] M. P. R. Waligorski, R. N. Hamm, R. Katz, The radial distribution of dose around the path of a heavy ion in liquid water, Nucl. Track. Radiat. Meas. 11 (1986) 309-319.

[55] M. P. Allen, D. J. Tildesley (Eds.), Computer Simulation of Liquid, Oxford University Press, England, 1987.

[56] D. S. Ivanov, L. V. Zhigilei, Combined atomistic-continuum modeling of short-pulse laser melting and disintegration of metal films, Phys. Rev. B 68 (2003) 064114-22.

[57] K. Nordlund, PARCAS computer code, private communication (2010). 
[58] D. W. Brenner, O. A. Shenderova, J. A. Harrison, S. J. Stuart, B. Ni, S. B. Sinnott, A second-generation reactive empirical bond order (REBO) potential energy expression for hydrocarbons, J. Phys.: Condens. Matter 14 (2002) 783-802.

[59] K. Nordlund, J. Keinonen, T. Mattila, Formation of ion irradiation induced small-scale defects on graphite surfaces, Phys. Rev. Lett. 77 (1996) 699-702. doi:10.1103/PhysRevLett.77.699.

URL http://link.aps.org/doi/10.1103/PhysRevLett.77.699

[60] J. F. Ziegler, J. P. Biersack, U. Littmark (Eds.), The Stopping and Range of Ions in Matter, Pergamon, New York, 1985.

[61] L. Lindsay, D. A. Broido, Optimized tersoff and brenner empirical potential parameters for lattice dynamics and phonon thermal transport in carbon nanotubes and graphene, Phys. Rev. B 81 (2010) 205441. doi:10.1103/PhysRevB.81.205441.

URL http://link.aps.org/doi/10.1103/PhysRevB.81.205441

[62] A. A. Balandin, S. Ghosh, W. Bao, I. Calizo, D. Teweldebrhan, F. Miao, C. N. Lau, Superior Thermal Conductivity of Single-Layer Graphene, Nano Letters 8 (3) (2008) 902-907, pMID: 18284217. arXiv:http:// dx.doi.org/10.1021/n10731872, doi:10.1021/n10731872.

URL http://dx.doi.org/10.1021/n10731872

[63] W. Cai, A. L. Moore, Y. Zhu, X. Li, S. Chen, L. Shi, R. S. Ruoff, Thermal Transport in Suspended and Supported Monolayer Graphene Grown by Chemical Vapor Deposition, Nano Letters 10 (5) (2010) 1645-1651, pMID: 20405895. arXiv:http://dx.doi.org/10.1021/ nl9041966, doi:10.1021/n19041966.

URL http://dx.doi.org/10.1021/n19041966

[64] C. Kittel, Introduction to solid state physics, Wiley, 2005.

[65] K. Kang, D. Abdula, D. G. Cahill, M. Shim, Lifetimes of optical phonons in graphene and graphite by time-resolved incoherent anti-stokes raman scattering, Phys. Rev. B 81 (2010) 165405. doi:10.1103/PhysRevB. 81.165405.

URL http://link.aps.org/doi/10.1103/PhysRevB.81.165405 
[66] J. Ziegler, J. Biersack, M. Ziegler, SRIM-2008.04 software package, available onlike at http://www.srim.org.

[67] B. Rethfeld, A. Rämer, N. Brouwer, N. Medvedev, O. Osmani, Electron dynamics and energy dissipation in highly excited dielectrics, Nucl. Instr. Meth. Phys. Res. B 327 (0) (2014) 78 - 88. doi:http://dx.doi.org/10.1016/j.nimb.2013.10.087.

URL http://www.sciencedirect.com/science/article/pii/ S0168583X14001554

[68] J. C. Johannsen, S. Ulstrup, F. Cilento, A. Crepaldi, M. Zacchigna, C. Cacho, I. C. E. Turcu, E. Springate, F. Fromm, C. Raidel, T. Seyller, F. Parmigiani, M. Grioni, P. Hofmann, Direct View of Hot Carrier Dynamics in Graphene, Phys. Rev. Lett. 111 (2013) 027403.

[69] G. Kresse, J. Furthmüller, Efficient iterative schemes for ab initio totalenergy calculations using a plane-wave basis set, Phys. Rev. B 54 (1996) $11169-11186$.

[70] K. Saito, J. Nakamura, A. Natori, Ballistic thermal conductance of a graphene sheet, Phys. Rev. B 76 (2007) 115409. doi:10.1103/ PhysRevB.76.115409.

URL http://link.aps.org/doi/10.1103/PhysRevB.76.115409

[71] S. Yigen, V. Tayari, J. O. Island, J. M. Porter, A. R. Champagne, Electronic thermal conductivity measurements in intrinsic graphene, Phys. Rev. B 87 (2013) 241411.

[72] G. F. Giuliani, J. J. Quinn, Lifetime of a quasiparticle in a twodimensional electron gas, Phys. Rev. B 26 (1982) 4421-4428. doi: 10.1103/PhysRevB . 26.4421. URL http://link.aps.org/doi/10.1103/PhysRevB.26.4421

[73] A. Principi, G. Vignale, Violation of the wiedemann-franz law in hydrodynamic electron liquids, Phys. Rev. Lett. 115 (2015) 056603. doi: 10.1103/PhysRevLett.115.056603.

URL http://link.aps.org/doi/10.1103/PhysRevLett.115.056603

[74] S. Fujii, M. Ziatdinov, M. Ohtsuka, K. Kusakabe, M. Kiguchi, T. Enoki, Role of edge geometry and chemistry in the electronic properties of 
graphene nanostructures, Faraday Discuss. 173 (2014) 173-199. doi: 10.1039/C4FD00073K.

URL http://dx.doi.org/10.1039/C4FD00073K

[75] Y. Wang, C. Grygiel, C. Dufour, J. Sun, Z. Wang, Y. Zhao, G. Xiao, R. Cheng, X. Zhou, J. Ren, et al., Energy deposition by heavy ions: Additivity of kinetic and potential energy contributions in hillock formation on caf2, Scientific reports 4.

[76] J. C. Meyer, F. Eder, S. Kurasch, V. Skakalova, J. Kotakoski, H. J. Park, A. Chuvilin, G. Benner, A. V. Krasheninnikov, U. Kaiser, S. Roth, S. Eyhusen, Accurate Measurement of Electron Beam Induced Displacement Cross Sections for Single-Layer Graphene, Phys. Rev. Lett. 108 (19) (2012) 196102. doi:10.1103/PhysRevLett.108.196102.

[77] A. Meftah, F. Brisard, J. Costantini, M. Hage-Ali, J. Stoquert, F. Studer, M. Toulemonde, Swift heavy ions in magnetic insulators: a damge-cross.section velocity effect, Physical Review B 48 (2) (1993) 920 .

[78] A. V. Krasheninnikov, F. Banhart, Engineering of nanostructured carbon materials with electron or ion beams, Nature Mat. 6 (2007) 723-733.

[79] T. Y. Kim, C.-H. Park, N. Marzari, The electronic thermal conductivity of graphene, Nano letters. 